\title{
The perspectives of molecularly targeted therapies which this issue illustrates
}

\section{Noriomi Matsumura ${ }^{1}$}

Published online: 8 June 2020

(C) The Japan Society of Clinical Oncology 2020

This issue contains four case reports of molecularly targeted therapy for cancer.

Hirose et al. investigated the association between HER2 expression and treatment course in a case of advanced gastric adenocarcinoma [1]. HER2 expression was not observed in endoscopic biopsies of the primary tumor. Tumors shrunk after S-1 with cisplatin treatment, and the patient underwent surgery. Interestingly, HER2 diffusely overexpressed in the resected surgical specimen. The disease was stable with trastuzumab-containing therapy for 6.4 months. This case might have intratumor heterogeneity with respect to HER2 positivity and the HER2-positive tumor cells might be refractory to chemotherapy. Thus, this case report suggests the importance of repeating the molecular analysis during the course of treatment.

The remaining three case reports relate to anti-PD-1 antibodies. Nonaka et al. reported a case in which anorectal malignant melanoma was resected but soon had distant metastasis and was treated with nivolumab [2]. Hayakawa et al. reported a case in which pembrolizumab was given to a metastatic urothelial carcinoma, and suffered myasthenia gravis [3]. In this case, pembrolizumab had a clear antitumor effect, but had to be discontinued because of the immunerelated adverse event (irAE). Hu et al. reported a case of acute kidney injury in a patient with gastric cancer treated with nivolumab [4]. Cessation of nivolumab and oral prednisolone administration rapidly improved the patient's renal function. In addition to the study of biomarkers to predict the susceptibility of immune checkpoint inhibitors, it is desirable to advance research to predict and manage irAE.

\section{References}

1. Hirose S, Moriwaki T, Yamaura M et al (2020) A case of advanced gastric cancer showing HER2 positivity after chemotherapy. Int Canc Conf J. https://doi.org/10.1007/s13691-020-00412-8

2. Nonaka K, Kudou K, Sasaki S et al (2020) Primary anorectal malignant melanoma with laparoscopic abdominoperineal resection: a case study and review of the relevant literature. Int Canc Conf J. https://doi.org/10.1007/s13691-020-00401-x

3. Hayakawa N, Kikuchi E, Suzuki S et al (2020) Myasthenia gravis with myositis induced by pembrolizumab therapy in a patient with metastatic urothelial carcinoma. Int Canc Conf J. https:// doi.org/10.1007/s13691-020-00408-4

4. Hu Q, Hasuda H, Ueki K et al (2020) Reintroduction of nivolumab in a patient with gastric cancer after improvement of nivolumabinduced acute interstitial nephritis: a case report. Int Canc Conf J. https://doi.org/10.1007/s13691-020-00418-2

Publisher's Note Springer Nature remains neutral with regard to jurisdictional claims in published maps and institutional affiliations.
Noriomi Matsumura noriomi@med.kindai.ac.jp

1 Faculty of Medicine, Kindai University, Osakasayama, Japan 